\begin{tabular}{c} 
International Journal of Engineering \& Technology, $7(2)(2018) 746-749$ \\
International Journal of Engineering \& Technology \\
SPC \\
Website: $\begin{array}{c}\text { ww. sciencepubco.com/index.php/IJET } \\
\text { doi: } 10.14419 / \text { ijet. } 7 \text { i } 2.9434 \\
\text { Research paper }\end{array}$ \\
\hline
\end{tabular}

\title{
Real-time logs in virtual data-center
}

\author{
Dr. Chalasani Srinivas * \\ Associate Professor Department of CSE Koneru Lakshmaiah Education Foundation, Vaddeswaram Guntur, India \\ *Corresponding author E-mail: drchsinuresearch@gmail.com
}

\begin{abstract}
Penetration testing is a specialized security auditing methodology where a tester simulates an attack on a secured system. The main theme of this paper itself reflects how one can collect the massive amount of log files which are generated among virtual datacenters in real time which in turn also posses invisible information with excessive organization value. Such testing usually ranges across all aspects concerned to log management across a number of servers among virtual data centers. In fact, Virtualization limits the costs by reducing the need for physical hardware systems. Instead, require high-end hardware for processing. In the real-time scenario, we usually come across multiple logs among VCenter, ESXi, a VM which is very typical for performing manual analysis with a bit more time-consuming. Instead of configuring secure-ids automatically in a Centralized log management server gains a powerful full insight. Along with using accurate search algorithms, fields searching, which includes title, author, and also content comes out of searching, sorting fields, multiple-index search with merged results simultaneously updates files, with joint results grouping automatically configures few plugs among search engine file formats were effective measures in an investigation. Finally, by using the Flexibility Network Security Monitor, Traffic Investigation, offensive detection, Log Recording, Distributed inquiry with full program's ability can export data to a variety of visualization dashboard which exactly needed for Log Investigations across Virtual Data Centers in real time.
\end{abstract}

Keywords: Centralized Log Investigates Server; Log Management; VDC Builds; Elk Stacks Working Process; Virtualization; Vsphere Components, RealTime Logs; Virtual Datacenter (Vsphere); Time-Based Analysis.

\section{Introduction}

The securely centralized log management scenario is a Virtual technology help to the data center real-time activities such as Data Access, modification and upload into the server.In This virtual datacenter (VDC) is a large amount of data used to access multiple server or system in the network through cable and wireless connection into a web interface. VMware is a software developing company, especially for providing various virtualization Enterprises platforms like Vsphere suited for the data center product. In this sphere suite, many components are ESXi, Vcenter, and Vsphere client and so on. Personal computer log analysis is a simple way to understand, but in a data center has many more servers and system connected to a network whether connected or not understand also complicates before, but commercial software information and event management tool are available, but we have to take an open-source component to build secure log management tool in this component are Elasticsearch and Riemann [monitors distributed system]. Some of the Security principles are used in log management server known your system, the principle of least privilege, Defence in depth, Protection is key, but detection is a must then Know your enemy forensics investigation is a process to successfully solve case scenario for hacking machines affected system like who, where, why, when this type of question solved. Network connection established through cable or wireless as knowing use sharing data locally or world because reducing timeconsuming hands to hand or computer to computer.

VMware VSphere combination the basic real hardware components assets beyond various computing systems and Keep lack of virtual components to the datacenter. The Virtualization process that discontinuity strong network Communication hardware, oper- ating system, and applications running. The VSphere virtualization VM(virtual machine), OS(operating system), Working application service never deeply strained through the boundaries require by finding on a real machine. The virtual correlative of machine components such as routers, switches and storage operations in a Virtual-infra that can reach the organization. The VSphere maintain large collections of infrastructure, such as CPUs, storage, and networking, as a unity and powerful operating environment, and also maintain the complexity of a data center.The VMware vSphere software stack is composed of the virtualization, management, and interface layers. VMware Vsphere purpose Virtualization translates datacenters into extensible, amass computing infrastructures Virtualization divided applications and information from the hard to get hardware infrastructure. No Cloud without Virtualization. Cloud service is infrastructure as a service in virtualization user to run data backup tool is for investigation data it makes some logs for investigation suspected log successful view on dashboard only.Multiple logs have generated ESXi server, client logs there has server local path (/var/log/logname.log) in an approach manual is typical than we forward logs to real-time log server through the agent pulls into the Log server. The server has best ways is time base and host-based analysis.

\section{Related work}

A log management server is a create bundles of open sources Linux packages used developed new secure Centralized Real-time logs in datacenter and gathering information about logs in a system (Windows, Linux), server (ESXi server), VCenter (manage and service provided to ESXi server) by the intercommunication port Forwarding agent like (the agent collects information, log 
path is given to severe weather checking running port and available UDP port forward in the same gateway only.). Centralized logs show the profile of each system and server multi-threaded, Elasticsearch, Logstash, Kibana, Redis, Riemann, Elasticsearchcurator, work together on an open source stack is known as Centralized log investigate the tool (Centralized log management server). This Tool is an end-to-end search, monitoring and visualization platform that you can use to investigate log file sources in real networks. VSphere Infrastructure components: VCenter, ESXi server, VSphere Desktop client and Web Client [1].

\begin{tabular}{|c|c|}
\hline VDC Component & Centralized log management server \\
\hline $\begin{array}{l}\text { A physical machine } \\
\text { with Windows } 2012 \\
\text { server[1] } \\
\text { VSphere Infrastruc- } \\
\text { ture: VCenter, ESXi } \\
\text { Server, Vsphere client, } \\
\text { VM[1] } \\
\text { Nxlog agent[1] }\end{array}$ & $\begin{array}{l}\text { - A physical Machine or Virtual } \\
\text { machine with Linux Operating } \\
\text { System better is Ubuntu lite[3] } \\
\text { - } \quad \text { Apache_lucene, Logstash, Redis, } \\
\text { ElasticSearch, Kibana, Riemann, } \\
\text { Curator[3] } \\
\text { - Nxlog serve connection[3] } \\
\text { - Java openjdk, Bro IDS }\end{array}$ \\
\hline
\end{tabular}

\section{Praposed model}

(A) VDC (ESXi, VCenter, VM) Log Manual Investigation: Manual Investigation takes time to single server log analysis, manage and check the weather is currently logged or not. Attackers and user trying failure logged in authentication in the server at the time logs will generate particular user-id and IP address to access our server then investigator trying to real time case checking start logs malicious attack is done based on particular time user and IP address based criminal use system identification and report, but manual analysis time duration more than one data in on the only ESXi server if taken multiple esxi, vm, center its take more day in Investigator easy ways to overcome the problem through use centralized log management scenario into we have few advantageous add into its take log form system, server port forward using client agents.

Example: Esxi logs

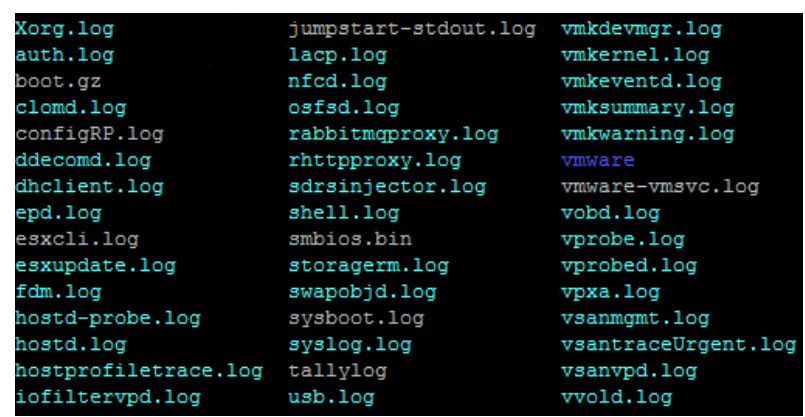

Fig. 1.0: Manual Analysis View All Logs in ESXI.

Vcenter logs and Vsphere Client logs are similar to that of esxi $\operatorname{logs}$ which is presented in the figure 1.0.

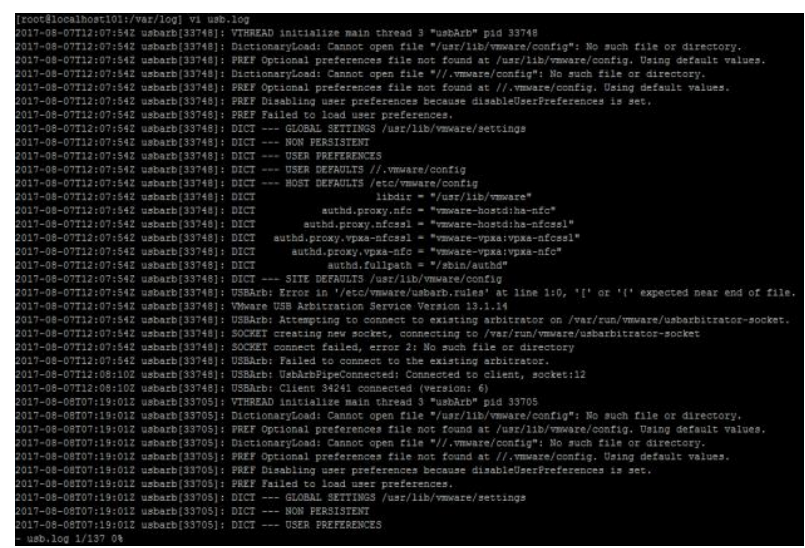

Fig. 2.1: Manual Analysis Single Esxi Logs.
(B) Centralized log management work Process (storage and index based Querying) [3]:

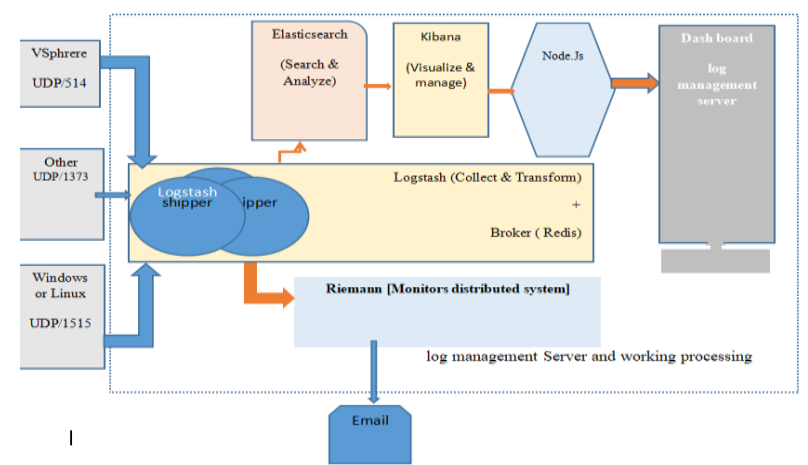

Fig. 2: Log Management Server and Working Processing.

- Logstash: Server-side data processing pipeline [1].

- ElasticSearch: Search engine based on Apache Lucene (Scalable, High-performance Indexing).

- Redis: In memory Data file system store, database, cache data, and message alerts broker.

- Kibana: Data visualization dashboard.

- Riemann: Riemann whole log events from your servers and applications with a dynamic stream processing language. Take time to Send an email for every exception in your application and system.

- Nxlog: A key concept used to collect logs from files (various formats) can be stored in files, forwarded to a remote $\log$ server.

- Node_js: Errorless for data-intensive at the same time, applications that run across made distribution apparatuses.

Figure (2) A log management server is an activity in real time collect remote system logs, store light-weight database for dataintensive distribution apparatuses is the node is filed. Logstash [11] is a data processing pipeline processing client to server port received data logs and sending mail to local database engine or a mail server support mail system on a network. Apache Lucene server (on Linux) performs fast searching index-based algorithm used on search engines (filtering logs) Filebeat system module executes the logs from visualizing the data, but broker all snipers are collecting and transform to searching is used Redis databases in the have cached data and message, alert. The above figure (2) shows the flow of the data process.

\section{Execution examination}

Diagram: Real-time search and visualization of log files in virtual data center Architecture.

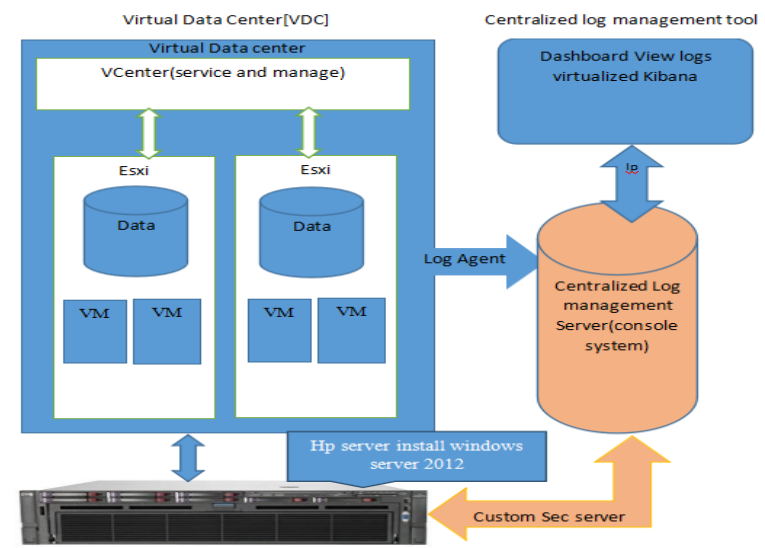

Fig. 3: Secure Centralized Log Management in Virtual Data-Center Architecture. 
Above Figure (3) Centralized log management tool (Investigate.) the performance fastest remote communication and execute query algorithms (apache-Lucene server) push to the index searching visualize presented in a dashboard integration and access to the wealth of details the agent functioning rapid access log forward through a computer protocol like UDP and TCP ports those types of logs are System, Server, VCenter, VSphere. Custom server development used components will be discussed resource above and configuration step also the main part of this project because network connection establishment performance single line connectivity done. There are many Advantage expose comparable Realtime $\log$ View on visualizing mode investigate multiple networks on the system. VMware provided investigation tool on ESXi only admin server access possible at one time, but data centers have multiple ESXi servers, VCenter, Virtual machines its take time more than one-day using VMware investigation tool. My proposed log management tool is access and investigation anywhere in a network, multiple logs, investigation start and identify few minute effected malware or unauthorized person in a network join manipulation check in-check out timestamp, message, alert to user administrate departments thought local mail and multiple packets hit count and sending access is possible or not analysis. It has a working process multiple accessed collects \& transforms data by searching analysis, display virtualizes management dashboard user web interface.

Deploy and configure:

Configuration Steps (connection to Centralize log management server) in a Virtual Datacenter [6]:

- Real-time logs Server using forward UDP 514 port to connect ESXi (data store, VM) in the server

- Real-time logs Server using forward SNMP 162 ports to connect VCenter (service \& management ESXi)

- VM Client System install and configure

The Nxlog agent to log forward though UDP 514,1515 Following below figures:
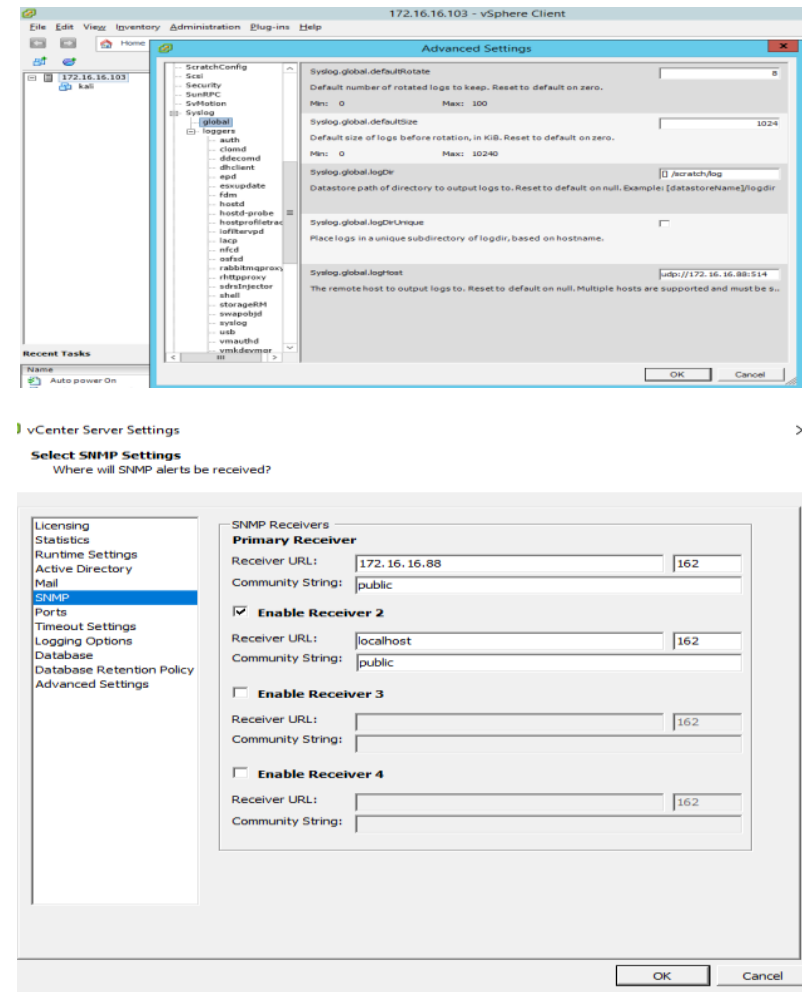

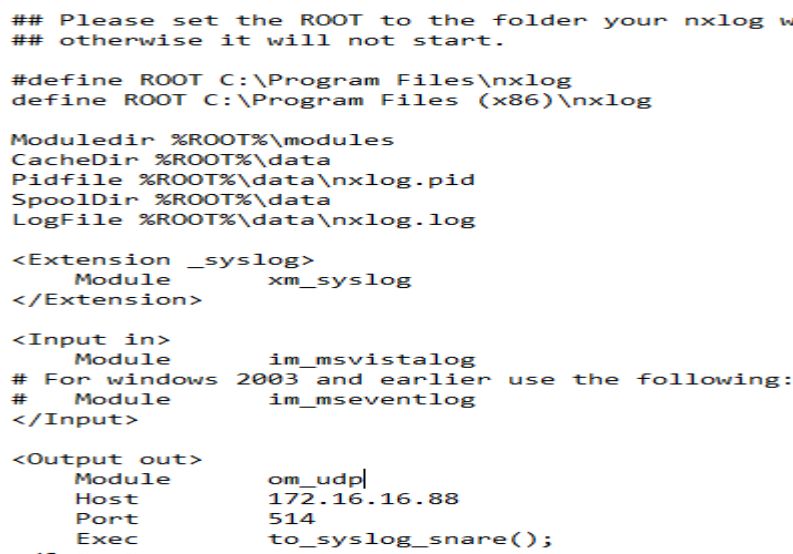

Fig. 4: Configuration Client System Using Agent Push

The above figure de script about the configuration step in clientside VM System (Windows, windows server or Linux) or physical machines(Windows, windows server or Linux) Nxlog agent download from the website installs the system and GOTO config file and check full fill connection change hostname is the log server IP address.

\section{Result analysis}

Event over time: This graph represents an event over time it is IPs hits count per 10s option are zoom in and zoom out graph bars, lines, stack, percent, legend and interval time auto, the $10 \mathrm{~s}, 1 \mathrm{~h}$ and month.

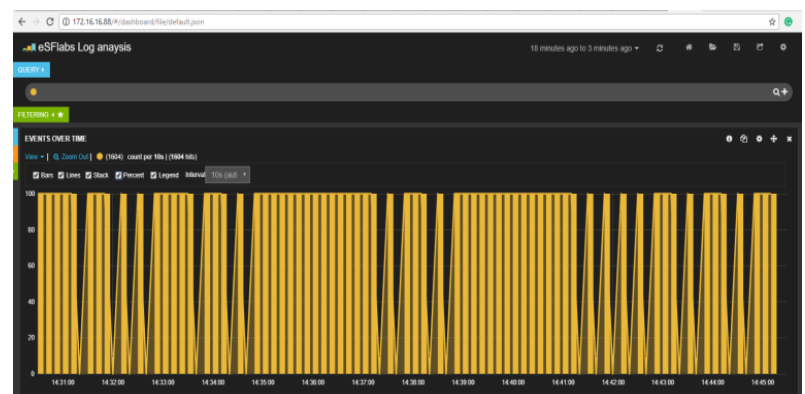

Fig. 5.1: Log Investigation Tool Web GUI

Time base analysis Particular IP: (172.16.16.94) IP address 53 per 10s@2017-08-16 17:00:20.

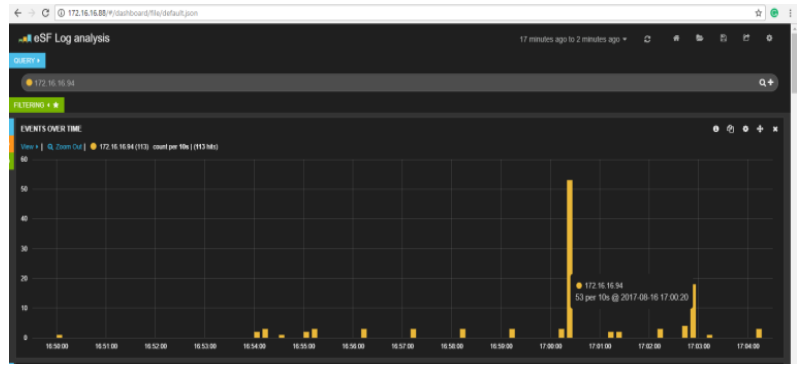

Fig. 5.2: Investigations on 172.16.16.94 (IP Address).

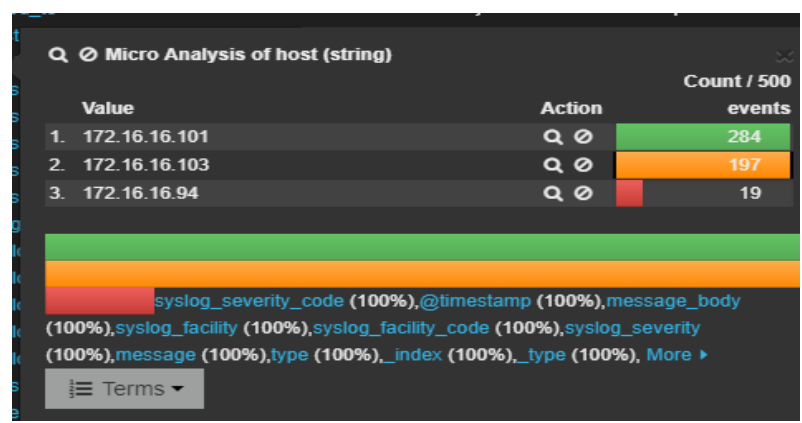




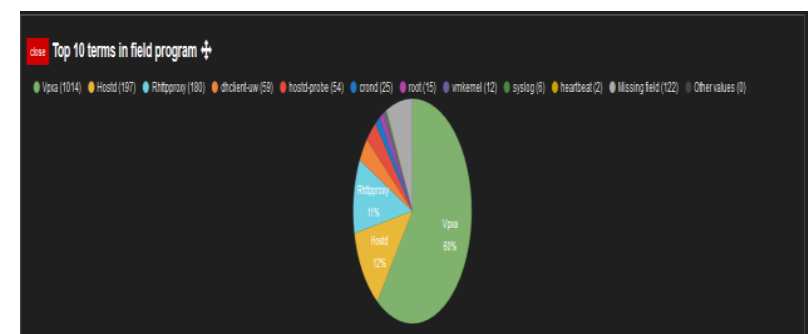

Fig. 5.3: Connected Client System and Top 10 Terms in Field.

The comprehensive test on each connects client system like ESXi server, center, and virtual machine. In this network explored different type of log alert and warning for data security for organization, network forensics evidence collect from different log only at the moment real-time view also importance security testing viewpoint.

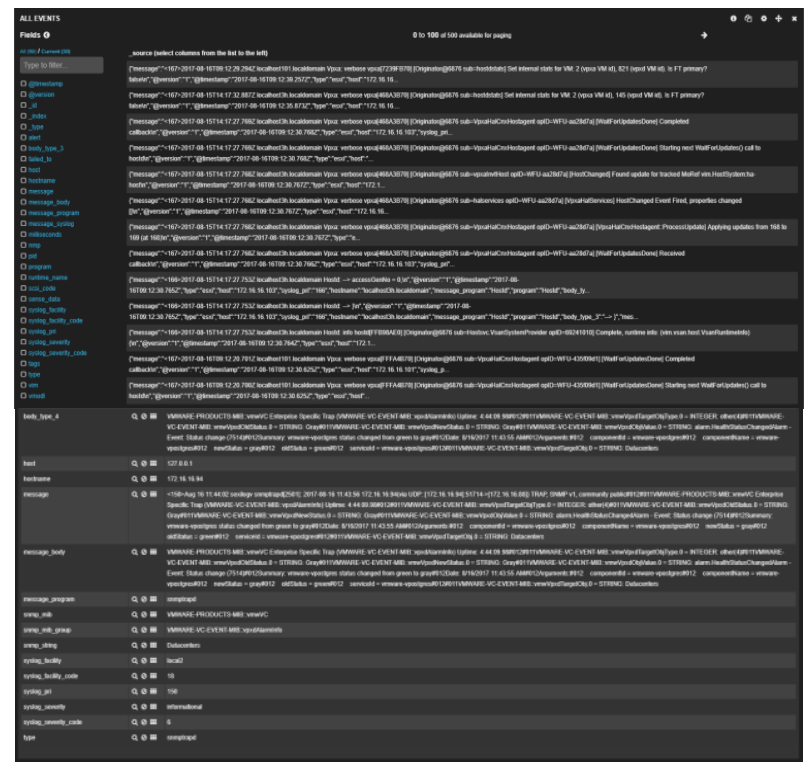

Fig. 5.5: Virtual Data Center Network Logs View on the.

The dashboard above figure exclusion result all Fields are left side check-box option, provide for choice particular field filler on a network and right-hand side source.

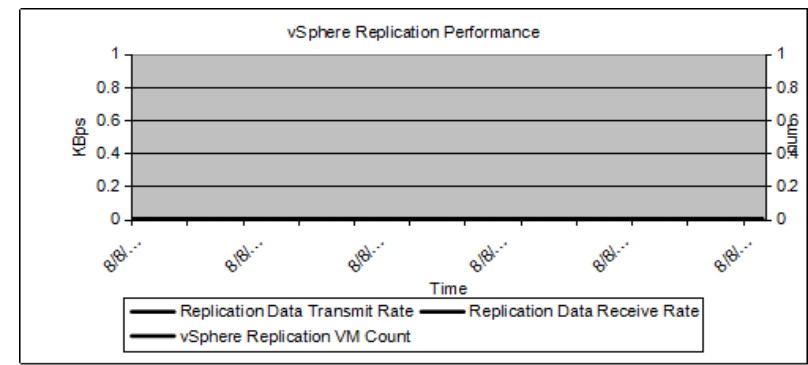

Fig. 6: Vsphere Replication Performance.

Above figure exploit Vsphere replication performance base on data transmit rate and receive data rate in a host hit packet collect and report to the administration.

\section{Conclusion}

This paper proposed immediate view of your network activity in Infrastructure Virtualization (VDC) service handling log export data to a variety of visualization dashboard and Organized log structure, manage to complicate in investigation The VSphere Component (VDC Component) have a lot of generated logs (ESXi server, VCenter, VM) service provide free of cost using open source Centralize log management server (investigation tool) is a visualize, monitor and index-based querying search. The perfor- mance results show that all logs in single visual monitor dashboard display successfully solves problems in a virtual data center.

\section{References}

[1] Author Peng Li,Lee Toderick,Joshua Noles "Provisioning Virtualized Datacenters Through Virtual Computing Lab" 2010.

[2] Forensics Log Invesigator(FLI)- a log analysis and Visulaization tool Thieu Van Tran Phan lowa State University 2007.

[3] Tarun Prakash,kritika patel "Geo-Identification of Web users through Logs using ELk stack" Project ieee papar 2016.

[4] Vmware White Paper "VMware Infrastructure Architecture Overview" [online] www: Vmware.com

[5] "The ELK Stack in a DevOps Environment" [online]: https://www.elastic.co/webinars/elk-stack-devops-environment.

[6] Ready-To-Log" virtual appliance made by community for community!.

[7] Feel free to follow SexiLog on"Building investigation tool"[online]:www.Sexilog.fr.

[8] Varun Kumar Manik, Deepak Arora "Performance Comparios of Commercial VMM: ESXI, XEN, HYPER-V \& KVM" india 2016.

[9] Zhijian Wang, Yanqin Zhu“A Centralized HIDS Framework for Private Cloud" jupan 2017

[10] Joshua Ojo Nehinbe"Log Analyzer for Network Forensics and Incident Reporting" ieee 2010.

[11] "riemman network monitoring and email forward "[online]:http://riemann.io/api/riemann.email.html.

[12] Pingkan P.i.Langi, Widyawan, Warsun Najib, Teguh Bharata Aji "An Evaluation of Twitter River and Logstash Performances as Elasticsearch inputs for Social Media Analysis of Twitter"IEEE 2015 https://doi.org/10.1109/ICTS.2015.7379895.

[13] Dong Nguyen Doan, Gabriel Iuhasz"Tunning Logstash Garbage Collection for High Throughput in a Monitoring Platform" IEEE.

[14] "Apache lucene fast string searching server help us Lucene Features" [online]: https://lucene.apache.org/core/.

[15] James Turnbull (Author) "The logstash book (Log management made easy)". 